\section{Microfiltración apical entre tres cementos utilizados en obturación retrógrada}

\section{Apical microfiltration between three cements used in retrograde obturation}

\section{Resumen}

Objetivo: Determinar la microfiltración apical de tres cementos utilizados en obturación retrógrada. Métodos: 45 piezas unirradiculares estandarizadas a $16 \mathrm{~mm}$ de longitud se instrumentaron con sistema ProTaper $\AA^{\circledR}$ manual e irrigaron con $\mathrm{NaClO}$ al 5,25\%. Las

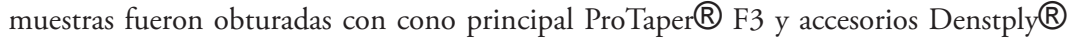
\#A y \#B; se realizó la recesión apical de $3 \mathrm{~mm}$, posteriormente se desobturó $3 \mathrm{~mm}$ con ultrasonido (Satelec Acteon) con punta s12/70D; se dividió la muestra en tres grupos para realizar obturación retrógrada con diferentes cementos de obturación cada uno. Las muestras se incubaron a $37^{\circ} \mathrm{C}$ por 3 días; se cubrieron con barniz de uñas excepto $2 \mathrm{~mm}$ apicales para ser sumergidas en azul de metileno al $1 \%$ por 48 horas, se lavaron y realizaron un corte longitudinal con disco de diamante \#2 para su análisis en el estereomicroscopio (EduBlue ED 1402S), con la ayuda de un micrómetro (Stainless Hardened) se obtuvieron diferentes valores de cada grupo, mismos que fueron analizados por medio de la comparación de medias y pruebas paramétricas. Resultados: La valoración media de la microfiltración que produce la obturación retrógrada indica que: el MTA® presentó

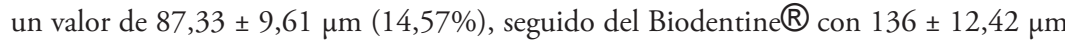
$(22,69 \%)$, con respecto al IRM® con mayor cantidad de microfiltración con un valor de $376 \pm 12,98 \mu \mathrm{m}(62,74 \%)$. Conclusiones: Al comparar las medias y porcentajes de

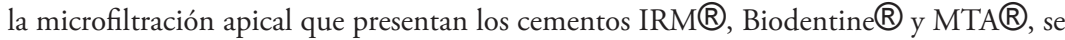
evidenció que el MTA® presentó el menor valor de microfiltración.

Palabras clave: Hipoclorito de sodio; Microfiltración; Obturación retrógrada.
ISSN: $1560-9111$

\section{Artículo Original}

Vinicio Vladimir Legarda Canales 1,a, Isadora Martini Garcia ${ }^{2, b}$, Paola Andrea Mena Silva 1,3,c, Viviana Marcela Hidalgo Moya 1,a, Raquel Esmeralda Guillén Guillén 1,b

${ }^{1}$ Universidad Central del Ecuador. Facultad de Odontología. Quito, Ecuador.

2 Universidade Federal do Rio Grande do Sul.

Faculdade de Odontologia. Porto Alegre, Brasil.

${ }^{3}$ Universidad Regional Autónoma de los Andes.

Facultad de Odontología. Ambato, Ecuador.

a Odontólogo(a).

${ }^{\mathrm{b}} \mathrm{PhD}$. en Odontología

c Especialista en Estética y Operatoria Dental.

\section{Correspondencia:}

Vinicio Vladimir Legarda Canales

Correo electrónico: deivisleg.88@hotmail.com

Conjunto Bosque Real. Torre B. Dep. 1D Av.

Occidental. Quito, Ecuador

\section{Coautores:}

Raquel Esmeralda Guillén Guillén raquelguillenguillen@gmail.com Isadora Martini Garcia isadora.garcia@ufrgs.br Paola Andrea Mena Silva pao_mena100@hotmail.com Viviana Marcela Hidalgo Moya vivihidalgo990@gmail.com

Conflicto de intereses: Los autores declaran no tener conflictos de interés.

Fuente de financiamiento: Autofinanciado.

Fecha de recepción: 21/11/18

Fecha de aceptación: 23/01/19

( ) Los autores. Este artículo es publicado por la revista Odontología Sanmarquina de la Facultad de Odontología, Universidad Nacional Mayor de San Marcos. Este es un artículo de acceso abierto, distribuido bajo los términos de la licencia Creative Commons Atribucion - No Comercia_Compartir Igual 4.0 Internacional. (http://creativecommons.org/licenses/by-nc-sa/4.0/) que permite el uso no comercial, distribución y reproducción en cualquier medio, siempre que la obra original sea debidamente citada. 
with the help of a micrometer (Stainless Hardened); different values of each group were obtained, then analyzed through comparison of means and parametric tests. Results: The average evaluation of the microfiltration produced by the retrograde filling indicates

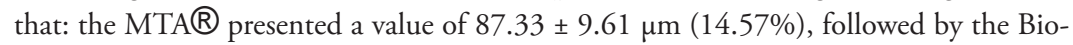

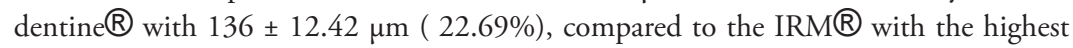
amount of microfiltration with a value of $376 \pm 12.98 \mu \mathrm{m}(62.74 \%)$. Conclusions: When comparing the means and percentages of the apical microfiltration of the IRM $®$, Biodentine $\AA$ and MTA $®$ cements; it was evident that the MTA $®$ showed the lowest microfiltration value.

Keywords: Microfiltration; Retrograde obturation; Sodium hypochlorite.

\section{Introducción}

El fracaso en el tratamiento endodóntico se encuentra vinculado a diversas causas, entre las que resaltan: la perforación del ápice, la transportación del conducto, la falta de reparación de una lesión apical y sobreobturación de la pieza, lo cual conlleva a la realización de una cirugía apical, la cual consiste en la exposición del ápice, realizando una resección radicular, para luego preparar una cavidad que será sellada de forma hermética, este procedimiento se lo conoce como obturación retrograda ${ }^{1}$. Uno de los factores más importantes es la correcta elección del material que se vaya a utilizar al momento de realizar este procedimiento, esta es una variable que nos permite saber qué cemento nos ofrece mejores resultados en un procedimiento de obturación retrógrada ${ }^{2,3}$. El material a elegir debe ser biocompatible, brindando un sellado hermético que imposibilite la microfiltración, no ser tóxico ni reabsorbible, radiopaco y bacteriostático ${ }^{3}$.

Es así que en el caso de los selladores apicales, tras una cirugía, el mineral trióxido agregado es el material mayormente empleado en campo, al poseer buena biocompatibilidad con los tejidos ${ }^{4,5}$. Un estudio realizado por Cedillo $e a l^{6}$, muestra que el Biodentine ${ }^{\circledR}$ (Septodont, Francia) es un excelente e innovador material por las propiedades mecánicas, de fácil manipulación y con una óptima biocompatiblidad indicado para restauraciones y procedimientos endodóncicos, así como también el MTA® (Angelus, Brasil) que es un cemento con propiedades para reparaciones apicales ${ }^{2}$.

Hoy en día el cemento de obturación retrógrada más utilizado es el MTA®, pero existe otro material con suficiente demanda que es el IRM® (Denstply Maillefer, Suiza), con la aparición del Biodentine ${ }^{\circledR}$ y sus excelentes características de biocompatibilidad se puede realizar comparaciones para evidenciar cuál de estos cementos presenta mayor efectividad ${ }^{7}$. Tal como lo menciona Aranguren ${ }^{8}$, el cual demostró que el MTA $®$ tiene similitud en las características al comparar con el super $\mathrm{EBA} \circledast$, sin embargo, presenta dificultades en la solubilidad con el transcurso del tiempo, estos cementos también son comparados con el IRM $囚$, y de igual manera el MTA® sigue siendo el ideal para la obturación retrógrada.
Debido a que estos materiales poseen propiedades físicas y químicas que difieren en cierta proporción; siendo uno superior que el otro, en aspectos que pueden influir en el desarrollo de la recuperación de tejidos periapicales; el objetivo de esta investigación es determinar cuál de estos tres cementos presentan un mejor sellado y menor microfiltración apical al ser utilizados en la obturación retrógrada.

\section{Métodos}

El protocolo de estudio fue aprobado por el Comité de Ética de la Universidad Central del Ecuador. Cuarenta y cinco piezas dentales unirradiculares fueron donadas para fines de investigación, la identidad e información de los pacientes donadores se mantiene en estricta confidencialidad. La selección de la muestra se realizó de manera aleatoria, se tomaron radiografías interproximales para verificar que las piezas cumplan con criterios de inclusión como: piezas con ápice formado, sin resorciones internas y raíces rectas de 0 a 5 grados según el ángulo de Schneider.

Las muestras se limpiaron con ultrasonido Woodpeker ${ }^{\circledR}$ con punta $\mathrm{G} 1$ con un nivel de intensidad 3 por 1 min para eliminar remantes de cálculo y tejidos. Se estandarizaron las piezas a $16 \mathrm{~mm}$ de longitud desde el ápice hasta la referencia coronal con una regla milimetrada. Se cortaron las coronas clínicas con un disco de diamante \#2 (JOTA $®)$.

Se realizó la preparación químico mecánica de los conductos con solución irrigante de hipoclorito de sodio al $5,25 \%$, para luego permeabilizar el conducto con una lima pre serie \#10 (Denstply Maillefer, Suiza). Seguido, se realizó la instrumentación de las muestras con el sistema manual ProTaper ${ }^{\circledR}$ (Denstply Maillefer, Suiza) hasta lima final \#F3 con técnica Crow Down acompañado de abundante irrigación y se secaron con puntas capillary.

A continuación se obturó con cemento endodóncico resinoso Adseal ${ }^{\circledR}$ y conos de gutapercha ProTaper ${ }^{\circledR}$ \# F3 como cono principal y conos accesorios \#A y \#B (Denstply Maillefer, Suiza), con técnica de condensación lateral; con la ayuda de un "guta cutter" se quemaron el sobrante de conos de gutapercha y compactaron para asegurar un buen sellado hermético. La entrada de los conductos fue sellada con Ionoseal $®$ (VOCO, Alemania). 


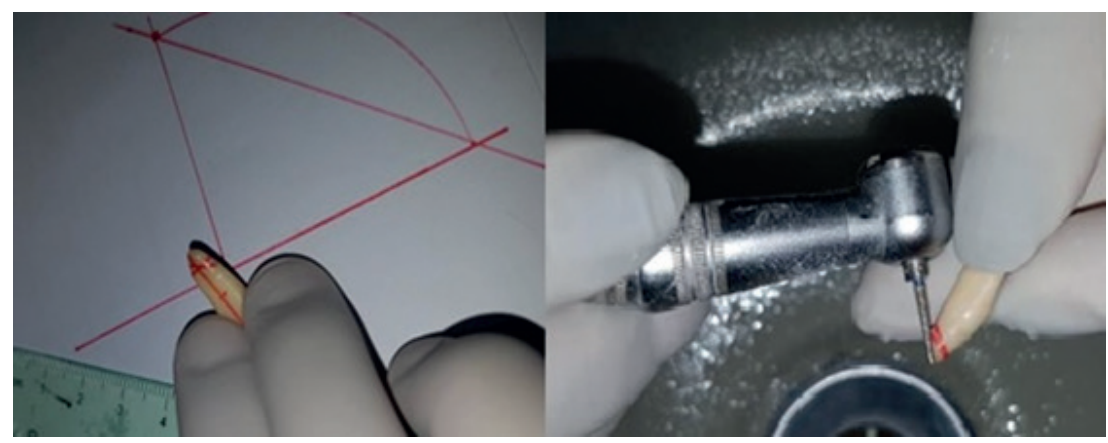

Figura 1. Medición de raíz para realizar apicetomía con corte perpendicular

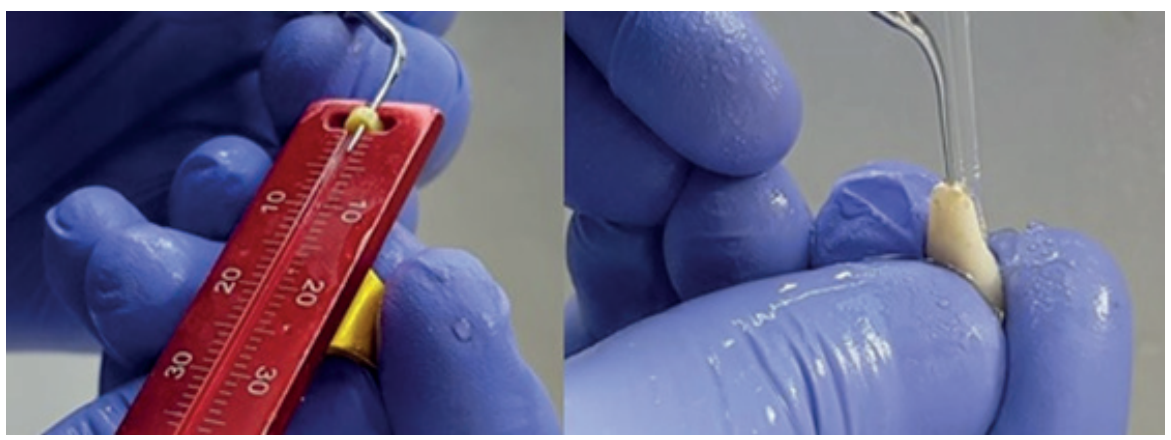

Figura 2. Desobturación con ultrasonido de $3 \mathrm{~mm}$ de gutapercha

Preparadas las muestras fueron divididas en tres grupos A, B y C de 15 piezas cada uno.

Apicectomía de las muestras: se realizó el corte de los 3 milímetros del tercio apical de las raíces con una fresa zecrya y contrángulo (NSK) a un ángulo de $90^{\circ}$. El corte perpendicular fue comprobado con la ayuda de un graduador (Fultons) (Figura 1).

Desobturación del ápice: una vez que se realizó el corte, se desobturó $3 \mathrm{~mm}$ con la ayuda de una punta ultrasónica s12/70D Satelec Acteon (Acteon ${ }^{\circledR}$, Francia) la cual ingresaron toda la longitud de su punta activa de $3 \mathrm{~mm}$ verificando con un tope de goma (Figura 2).

Obturación retrógrada de las muestras: la preparación de los cementos $\left(A: I R M \circledast\right.$, B: Biodentine ${ }^{\circledR}$ y C: $M T A \circledast$,) se realizó de acuerdo con las especificaciones del fabricante.

Las muestras se almacenaron en solución salina estéril en una estufa incubadora Hera Therm (Thermo Fisher Scientific ${ }^{\mathrm{TM}}$, USA) por 7 días a $37^{\circ} \mathrm{C}$ y $100 \%$ de humedad para incitar el fraguado de los cementos. A cada grupo se le asignó un color de barniz de uñas (Masglo) dejando libre $2 \mathrm{~mm}$ del ápice para realizar el proceso de filtración apical. Las muestras fueron sumergidas en tubos de ensayos con azul de metileno al 1\% (preparado en el laboratorio de química de la Facultad de Química de la Universidad Central del Ecuador) por 48 horas. Se realizó un corte longitudinal con un disco de diamante \# 2 (Jota) en las muestras para luego llevarlas al estereomicroscopio EduBlue ED 1402S (Euromex, Holanda) y medir las muestras con un micrómetro Stainless Hardened (Shenzhen Mantimelong Electro- nic, China). Una vez que los datos se recolectaron y verificaron se procedieron a exportarlos a un programa estadístico SPSS versión 23 para el respectivo análisis, en donde se utilizó la comparación de medias con pruebas paramétricas ANOVA y t de Student a un nivel de significancia del 5\%.

\section{Resultados}

Al comparar el grado de microfiltración de las muestras de cada grupo se demuestra que el MTA®, presenta valores menores entre 70-100 $\mu \mathrm{m}$, seguido de Biodentine ${ }^{\circledR}$ con un rango de 150 a $180 \mu \mathrm{m}$, y con mayor tendencia de microfiltración lo muestra el IRM $囚$ reportando un rango de 360 a $390 \mu \mathrm{m}$.

Respecto a los valores de microfiltración que generó el sellado de los tres cementos, se evidenció que el MTA® exhibió el menor valor de microfiltración y por lo tanto el menor porcentaje (14,57\%), mientras el Biodentine ${ }^{\circledR}$ obtuvo un $22,69 \%$ y el mayor valor reportado de microfiltración lo exhibe el IRM® con $62,74 \%$, de acuerdo a la cantidad de piezas que presentaron microfiltración.

En la tabla se presenta las medias obtenidas de cada grupo; el $p<0,05$, aprueba que existe diferencia entre la microfiltración apical que presentan los cementos IRM®, Biodentine $\AA$ y $M T A \circledast$ en la obturación retrógrada.

\section{Discusión}

El objetivo del presente estudio fue comparar in vitro el sellado apical entre tres cementos de obturación retrograda IRM $₫$, Biodentine $®$ y MTA $®$ mediante la penetración de azul de metileno $1 \%$. 
Tabla. Valores descriptivos de la microfiltración de los tres cementos

\begin{tabular}{|c|c|c|c|c|c|c|c|c|}
\hline \multirow{2}{*}{ Descripción } & \multirow{2}{*}{$\mathbf{n}$} & \multirow{2}{*}{ Media } & \multirow{2}{*}{ Desviación estándar } & \multirow{2}{*}{ Error estándar } & \multicolumn{2}{|c|}{$\begin{array}{l}95 \% \text { del intervalo de confianza para } \\
\text { la media }\end{array}$} & \multirow{2}{*}{ Mínimo } & \multirow{2}{*}{ Máximo } \\
\hline & & & & & Límite inferior & Límite superior & & \\
\hline IRM® & 15 & 376 & 12,98 & 3,35 & 368,81 & 383,19 & 360 & 390 \\
\hline Biodentine ${ }^{\circledR}$ & 15 & 136 & 12,42 & 3,20 & 129,12 & 142,87 & 120 & 150 \\
\hline MTA® & 15 & 87,33 & 9,61 & 2,48 & 82,01 & 92,65 & 70 & 100 \\
\hline Total & 45 & 199,77 & 128,12 & 19,09 & 161,28 & 238,27 & 70 & 390 \\
\hline
\end{tabular}

La microfiltración apical se da por la interfase diente material de obturación, esto puede evitarse a través de un sellado eficiente de las paredes de la cavidad pulpar 9. Para tal fin se debe seleccionar el mejor material de obturación retrógrada, que disminuye la capacidad de microfiltración apical e inhiba el desplazamiento de microorganismos infecciosos desde y hacia los tejidos periapicales, previniendo así futuras reinfecciones ${ }^{2}$.

Es por esta razón que se determinó la microfiltración apical con tres cementos utilizados en obturación retrógrada, resultando que el MTA® presentó menor valoración de microfiltración $(87,33 \pm 9,61 \mu \mathrm{m})$, lo que significa una disminución del azul de metileno dentro de las cavidades retrógrada (14,57\%), en comparación con el IRM® $(62,78 \%)$ y Biodentine $®(22,69 \%)$. Con el análisis de los resultados se demuestra que el MTA® permitió un mejor sellado, en contraste con el IRM® $(376 \pm 12,98 \mu \mathrm{m})$ y Biodentine ${ }^{\circledR}(136 \pm 12,42 \mu \mathrm{m})$, también se aprueba la hipótesis que establece que existe diferencia entre la microfiltración apical que presentan los cementos IRM $囚$, Biodentine $₫$ y MTA $®$ en la obturación retrograda $(\mathrm{p}<0,05)$, evidenciando que los valores de volumen de microfiltración difieren.

Estos hallazgos concuerdan con lo investigado en el 2015 por Mandava et al. ${ }^{10}$ donde estudiaron el comportamiento de microfiltración del Biodentine ${ }^{\circledR}(1170,96$ $\mu \mathrm{m})$ y $\mathrm{MTA} \circledast(321,23 \mu \mathrm{m})$ en obturación retrógrada, exhibiendo una menor microfiltración en el MTA® con una diferencia de 849,73 $\mu$ m. Además, hubo una diferencia estadísticamente significativa entre el grupo del MTA® y los grupos restantes.

Otros estudios que muestran similitud con los resultados de la presente investigación son los de Pereira et al. ${ }^{12}$, Erkut et al. ${ }^{13}$, Shahriar et al. ${ }^{14}$ y Ozbay et al. ${ }^{15}$ los cuales demostraron que el MTA® presentó un mejor sellado que otros materiales de relleno retrógrados y menor volumen de microfiltración apical. La justificación de ello es que el MTA® brinda un mayor sellado por la ligera expansión del material al entrar al conducto radicular y por la formación de cristales parecidos a la hidroxiapatita en la interfaz entre el material y la pared del conducto, debido a lo cual el material muestra una mejor adhesión que impide la penetración del tinte y por lo tanto exhibe una mínima microfiltración ${ }^{10,16}$.

En el 2009 Caballero et al. ${ }^{17}$ estudiaron tres materiales de obturación temporal en un estudio in vitro y determinaron que el IRM ${ }^{\circledR}$ presentó el mayor nivel de microfiltración en contraste con los demás materiales de obturación de la investigación. Estos hallazgos concuerdan con los del presente estudio, donde el IRM® exhibió el mayor porcentaje de microfiltración apical (62,78\%). Sobre esto, en el 2012 Grech et al. ${ }^{18}$ manifiestan que el IRM® estaba compuesto de óxido de zinc y una fase rica en carbono. A través de un análisis de espectroscopia de dispersión de rayos X, identificaron que el IRM® está mezclado con eugenol, producto orgánico y contiene grupos de alcohol y metoxi que se detectaron en el escáner espectrofotómetro de infrarrojo. Demostrando que existían áreas muy grandes del eugenol presentes en el material fraguado de acuerdo a las micrografías electrónicas de barrido. Esto induce a establecer que por esta área puede migrar mayor cantidad de fluido y disminuir la eficiente obturación retrógrada de este material. La selección clínica del IRM $®$ puede basarse más en esta propiedad biológica que en la capacidad de sellado.

Los resultados del estudio de Kokate y Pawar en el 2012 ${ }^{19}$ no concuerdan con los de la presente investigación, ellos demostraron que el Biodentine ${ }^{\circledR}(130 \pm 60 \mu \mathrm{m})$ presentó una menor microfiltración en comparación con el MTA® $(73 \pm 130 \mu \mathrm{m})$. Mencionan que se debe a la modificación composicional del Biodentine®, por la adición de aceleradores y suavizantes de fraguado, una nueva formulación de cápsula pre dosificada para usar en un dispositivo de mezclado que mejora en gran medida las propiedades físicas del material.

De acuerdo a Cedrés et al. ${ }^{20}$ el Biodentine ${ }^{\circledR}$ es similar en la composición básica e indicaciones endodónticas al MTA $®$, los fabricantes del Biodentine ${ }^{\circledR}$ afirman que está constituido de polvo modificado (de silicato tricálcico con adición de carbonato de calcio como relleno y óxido de circonio) y esto mejora el sellado del material. Esta explicación justifica que en el presente estudio se identificó el Biodentine ${ }^{\circledR}$ como el segundo material de obturación que exhibió menor microfiltración del azul de metileno $(136 \pm 12,42 \mu \mathrm{m})$, con una diferencia entre el MTA® de 48,67 $\mu \mathrm{m}$, además es necesario considerar que estadísticamente sí existió diferencia significativa entre la valoración de la microfiltración del MTA® y Biodentine ${ }^{\circledR}$. Es recomendable seguir las instrucciones del fabricante de los cementos, sin improvisar, para evitar que durante la manipulación se cometan errores que altere los valores de microfiltración de los cementos en obturación retrógrada. 


\section{Referencias bibliográficas}

1. Aguilar GE, García ARL. Estudio comparativo in vitro para medir la microfiltración en obturación retrógrada con PRO ROOT ${ }^{\oplus}, \mathrm{CPM}^{\oplus}$ y Súper-EBA ${ }^{\oplus}$. Rev Odont Mex. 2007;11(3):140-144.

2. Díaz A, Ramos J, Arrieta L. Apicectomia con obturación retrograda e injerto óseo para el tratamiento de una lesión apical. Duazari. 2010;7(2):228-233.

3. Silva D, Herz F, Rodríguez F, González L. Evaluación de la microfiltración apical de Biodentine ${ }^{\mathrm{TM}}$ como material de obturacion apical mediante el transporte de fluidos computarizados. Rev ADM. 2016;72(2):65-71.

4. Butt N, Talwar S, Chaudhry S, Roongta N, Ruchika YS, Bali A. Comparison of physical and mechanical properties of mineral trioxide aggregate and Biodentine. Indian J Dent Res. 2014;25(6):692-7.

5. Cohen S, Heargreaves K, Berman L. Vías de la Pulpa. 10th ed. Barcelona: Elsevier; 2011. p. 1104

6. Cedillo J, Espinosa R, Curiel R, Huerta A. Nuevo sustituto bioactivo de la dentina; silicato tricalcico purificado. Rodyb. 2013;2(2):1-12.

7. Correa M, Castrillón N. Comparación de microfiltración apico-coronal entre MTA y Biodetine en dientes unirradiculares. Odontoinvestigación. 2015;1(1):1-5.

8. Aranguren J. Materiales para la obturación a retro: de la amalgama al agregado de mineral trioxido (MTA). Gaceta Dental. 2009;(17):1-16.

9. Monardes H, Abarca J, Castro P. Microfiltración Apical de Dos Cementos Selladores. Un Estudio in vitro. Int. J Odontosmat. 2014;8(3):393-398.

10. Mandava P, Bolla N, Thumu J, Vemuri S, Chukka S. Microleakage evaluation around retrograde filling materials prepared using conventional and ultrasonic techniques. J Clin Diagn Res. 2015;9(2):43-46.
11. Torabinejad M, Hong C, McDonald F, Pitt Ford T. Physical and chemical properties of a new root-end filling material. J Endod. 1995;21(7):349-353.

12. Pereira C, Cenci M, Demarco F. Sealing ability of MTA, Super EBA, Vitremer and amalgam as root-end filling materials. Braz Oral Res. 2004;18(4):317-21.

13. Erkut S, Tanyel C, Keklikoglu N, Yildirim S, Katboglu A. A Comparative Microleakage Study of Retrograd Filling Materials. Tubitak. 2006;36(1):113-120.

14. Shahriar S, Hamid Y, Saeed R, Mahsa E, Sahar S, Mahsa $\mathrm{U}$. Comparison of the sealing ability of mineral trioxide aggregate and Portland cement used as root-end filling materials. J Oral Sci. 2011;53(4):517-522.

15. Ozbay G, Kitiki B, Peker S, Kargul B. Apical Sealing Ability of a Novel Material: Analysis by Fluid Filtration Technique. Acta Stomatol Croat. 2014;48(2):132-139.

16. Espir C, Guerreiro J, Spin R, Chávez G, Berbert F. Solubility and bacterial sealing ability of MTA and root-end filling materials. J Appl Oral Sci. 2016;24(2):121-125.

17. Caballero C, García C, Untiveros G. Microfiltración coronal in vitro con tres materiales de obturación temporal utilizados en endodoncia. Rev Estomatol Herediana. 2009;19(1):27-30.

18. Grech L, Mallia B, Camilleri J. Characterization of set Intermediate Restorative Material, Biodentine, Bioaggregate and a prototype calcium silicate cement for use as root-end filling materials. J Endod. 2013;46(7):632-641.

19. Kokate S, Pawar A. An in vitro comparative stereomicroscopic evaluation of marginal seal between MTA, glass inomer cement $\&$ biodentine as root end filling materials using 1\% methylene blue as tracer. Endodontology. 2012;24(2):36-42.

20. Cedrés C, Giani A, Laborde J. Una Nueva Alternativa Biocompatible: BIODENTINE. Actas Odontológicas. 2014;11(1):11-16. 
